Educa UMCH. Revista sobre Educación y Sociedad, 2020, 1(15), 5-22.

https://doi.org/10.35756/educaumch.v1i15.135

\title{
Análisis Psicométrico Preliminar de la escala Perfil de Impacto Emocional COVID-19 en Universitarios Peruanos
}

Preliminary Psychometric Analysis of the Emotional Impact Profile scale COVID-19 in Peruvian University Students

Recibido el 20/ 03/ 2020. Revisión del 03/02 al 25/ 04/ 2020. Aceptado 28/05/2020

Luis Rodríguez de los Ríos

(ORCID: 0000-0002-2274-695X)

rodri0351@hotmail.com

Universidad Nacional de Educación Enrique Guzmán y Valle. Lima-Perú

Alberto Quintana Peña

(ORCID: 0000-0003-4305-137X)

albertolqp@gmail.com

Universidad Nacional Mayor de San Marcos. Lima, Perú

Jaime Aliaga Tovar

(ORCID: 0000-0002-4091-3205)

aliato1@hotmail.com

Universidad Nacional Mayor de San Marcos. Lima, Perú

\author{
Brian Norman Peña-Calero \\ (ORCID: 0000-0002-1073-9306) \\ brianmsm@gmail.com
}

Grupo de Estudios Avances en Medición Psicológica, Universidad Nacional Mayor de San Marcos. Lima, Perú 


\section{Resumen}

Se analiza y establece en este estudio de tipo instrumental (Ato, López-García, \& Benavente, 2013), las cualidades psicométricas fundamentales del Perfil de Impacto Emocional COVID-19 (García Pérez \& Magaz, 2020), con la finalidad de utilizarlo a posteriori en la población de estudiantes universitarios con relación al impacto emocional generado por los efectos de este virus en la población de Lima. En una muestra de 161 estudiantes universitarios (117M; $44 \mathrm{~V}$ ) de una edad promedio de 23 años $(R \mid Q=3)$, se administró un formulario virtual del Perfil, estimándose la validez analizando su estructura interna empleando un análisis factorial confirmatorio (AFC), intercorrelacionando los ítems de las cinco escalas del Perfil: ira/resentimiento, miedo, ansiedad, estrés y depresión, con el coeficiente policórico y empleando el estimador Weighted Least Square Mean and Variance Adjusted (WLSMV). Los índices resultantes muestran un ajuste empírico tipificado de excelente entre la estructura teórica del Perfil y los datos colectados $\left(X^{2}=426.83, \mathrm{gl}=265, \mathrm{CFI}=.970, \mathrm{TLI}=.966\right.$, RMSEA $=.062$, SRMR $=.079)$. Por otro lado, la fiabilidad de las cinco escalas calculadas por los coeficientes alfa y omega, arrojaron resultados elevados (>.85). Estas excelentes cualidades psicométricas avalan el uso del Perfil en procesos de investigación sobre efectos emocionales del COVID-19.

Palabras Clave: Covid-19, Impacto emocional, estudiantes universitarios.

\section{Summary}

The instrumental psychometric qualities of the Emotional Impact Profile COVID-19 (García Pérez \& Magaz, 2020) are analyzed and established in this instrumental study (Ato, López-García, \& Benavente, 2013), in order to use it posteriori in the university student population in relation to the emotional impact generated by the effects of this virus in the Lima population. In a sample of 161 university students (117M; 44V) of an average age of 23 years $(R I Q=3)$, a virtual Profile form was administered, estimating the validity by analyzing its internal structure using a confirmatory factor analysis (AFC), intercorrelating the items of the five scales of the Profile: anger / resentment, fear, anxiety, stress and depression, with the polychoric coefficient and using the Weighted Least Square Mean and Variance Adjusted (WLSMV) estimator. The 
resulting indices show an excellent empirical typified fit between the theoretical structure of the Profile and the data collected $\left(X^{2}=426.83, \mathrm{gl}=265, \mathrm{CFI}=.970, \mathrm{TLI}=\right.$ $.966, \mathrm{RMSEA}=.062, \mathrm{SRMR}=.079)$. On the other hand, the reliability of the five scales calculated by the alpha and omega coefficients, yielded high results (> .85). These excellent psychometric qualities endorse the use of the Profile in research processes on emotional effects of COVID-19.

\section{Introducción}

La pandemia del COVID-19 es actualmente el principal problema en salud pública a nivel mundial, registrando a la fecha a más de 12320000 casos confirmados y una cifra de fallecimientos superior a 556000 personas a la fecha (World Health Organization, 2020). La ausencia de un tratamiento específico y efectivo (Harapan et al., 2020), ha llevado a los gobiernos a realizar esfuerzos por frenar la expansión de la enfermedad mediante la toma de diversas medidas reguladoras obligatorias como la distancia social, uso de mascarillas, establecimiento de estados de emergencia y la puesta de cuarentena a fin de restringir el contacto social de las personas (Lin et al., 2020; Qarnain, Muthuvel, \& Bathrinath, 2020).

Las políticas de gobierno implementadas han ido cambiando conforme pasan los meses, sustentándose muchas de ellas en estudios científicos y modelamientos matemáticos de la evolución de la enfermedad (Huamaní, Timaná-Ruiz, Pinedo, Pérez, \& Vásquez, 2020). Sin embargo, el impacto positivo de las medidas tomadas es de difícil valoración en comparación a los efectos adversos provocados, motivo por el que algunos países han desistido de implementar las medidas mencionadas.

La medida con mayor impacto tanto positivo como negativo en este contexto es la cuarentena obligatoria (Sánchez-Duque, Arce-Villalobos, \& Rodríguez-Morales, 2020). Los países que la han implementado han obtenido una menor tasa de contagio de la enfermedad (Shen et al., 2020), pero a la vez consecuencias económicas tanto para las grandes y pequeñas empresas, como para la población trabajadora (Ashraf, 2020). Es necesario considerar que los países con menor recursos económicos, como 
es el caso de América Latina, presentan una capacidad de reacción menor para hacer frente a la enfermedad (Sánchez-Duque et al., 2020), y sus consecuencias.

El impacto psicológico es otro factor de suma importancia que se encuentra relacionado con la enfermedad del COVID-19 y situación de cuarentena en personal de salud (Pappa et al., 2020), en quien la padece (Guo et al., 2020), sus familiares (Sivashanker et al., 2020), y personas en general (Huarcaya-Victoria, 2020), que podrían estar en riesgo de contagiarse o relacionado de alguna manera con la propagación de la enfermedad. Entre las categorizaciones más comunes con respecto al impacto en la salud mental de las personas se encuentra los desórdenes de pánico, ansiedad, depresión (Wang, Wen, Liu, Dong, \& Cui, 2020), estrés, autolesiones (Holmes et al., 2020), frustración, ira y miedo al contagio (Brooks et al., 2020).

La situación del COVID-19 también ha generado un impacto psicosocial importante en la población (Dubey et al., 2020), al estar expuestos constantemente a mensajes gubernamentales sobre reglamentos y regulaciones, noticias de los medios de comunicación y sobre información sobre la situación. Se ha encontrado relación entre la salud mental afectada durante el contexto del COVID-19 y el debilitamiento del sistema inmunológico (Troyer, Kohn, \& Hong, 2020), que podría debilitar la oportunidad de las personas de afrontar a la enfermedad.

La población joven, a pesar de ser menos propensa a tener una situación agravante a nivel físico producto del contagio de la enfermedad, no está exenta de que su salud mental se vea afectada con la aparición de cuadros de estrés post-traumático, desórdenes de depresión y ansiedad, incluyendo la propensión a la ideación suicida y uso de drogas (Guessoum et al., 2020). Esta situación está estrechamente relacionada con la caracterización descrita acerca de la salud mental en jóvenes universitarios reportado a lo largo de diferentes investigaciones (Chau \& Vilela, 2017; Cova Solar et al., 2007; Gutiérrez Rodas et al., 2010; Micin \& Bagladi, 2011; Neponuceno et al., 2019; Perales et al., 2019); y que se encuentra a su vez relacionado con su desenvolvimiento universitario y rendimiento académico (Feldman et al., 2008; Palacio Sañudo, Martínez de Biava, Ochoa, \& Tirado Muñoz, 2006; Suárez-Montes \& Díaz-Subieta, 2015). 
De esta manera, disponer de herramientas que permitan recolectar información de calidad acerca de las afecciones en la salud mental producto de la pandemia del COVID-19 es una necesidad primordial (Holmes et al., 2020). Los instrumentos breves pueden brindar información valiosa en corto tiempo y permitir que su aplicación sea masiva fácilmente. Así, un instrumento de medición podrá tener la función de recolectar información necesaria e importante sobre la población general a fin de focalizar esfuerzos e intervenciones en quiénes más lo necesitan.

Así, el objetivo de la investigación es realizar un análisis psicométrico preliminar de un instrumento para medir el impacto emocional producto del COVID-19 en la población general.

\section{Metodología}

\section{Diseño y participantes}

La investigación se enmarca en un enfoque cuantitativo mediante el diseño de investigación instrumental (Ato, López-García, \& Benavente, 2013), debido a que se analizan las propiedades psicométricas de un instrumento de medición en población general.

Los participantes de la investigación estuvieron conformados por 161 estudiantes universitarios con una edad promedio de 23 años $(R \mid Q=3)$, de los cuales 117 fueron del género femenino y 44 , del masculino.

\section{Instrumento}

El instrumento utilizado es el Perfil de Impacto Emocional de la COVID19 (PIE COVID19; García Pérez \& Magaz, 2020), creado en España para la evaluación en población general. El instrumento se compone de las 5 categorizaciones más comunes a modo de dimensiones con 5 ítems cada uno y con alternativas de respuesta uniforme en los ítems que pertenecen a una misma dimensión, pero diferenciadas entre las distintas dimensiones. Las alternativas de respuesta fueron puntuadas de 0 a 3 de acuerdo a las alternativas en cada dimensión: 
1. Dimensión Ira/Resentimiento: No me enfada, me enfada algo, me enfada bastante y me enfada mucho.

2. Dimensión Miedo: Nada de miedo, algo de miedo, bastante miedo y mucho miedo.

3. Dimensión Ansiedad: Casi nunca, algunas veces, a menudo y constantemente.

4. Dimensión Estrés: Casi nunca, algunas veces, a menudo y constantemente.

5. Dimensión Depresión: Casi nunca, algunas veces, a menudo y constantemente.

\section{Procedimientos para recolección de información y análisis de datos}

Se elaboró un formulario virtual por medio de la herramienta de Formularios de Google donde se plasmaron algunos datos sociodemográficos a solicitar y la respuesta a cada uno de los ítems de acuerdo a las alternativas de respuestas. Los ítems registrados en el formulario se configuraron para ser contestadas de forma obligatoria. En el formulario se informó el motivo del estudio y se dio la opción de aceptar la recolección de datos o rechazarla (4 personas rechazaron).

Los datos fueron almacenados en una hoja de cálculo para su análisis posterior. Se realizó la limpieza de datos uniformizando información. Posterior a ello se realizó un análisis descriptivo de los ítems a fin de verificar la variabilidad de respuesta de las personas a los reactivos. Luego, se buscó brindar evidencia de validez basada en la estructura interna de la prueba mediante el análisis factorial confirmatorio (AFC). Este análisis se ejecutó teniendo en cuenta la característica de la ordinalidad de los ítems (correlaciones policóricas) y uso del estimador Weighted Least Square Mean and Variance Adjusted (WLSMV). La fiabilidad por el método de consistencia interna fue estimada mediante el coeficiente alfa y omega a partir del modelo factorial evaluado. 


\section{Resultados}

Análisis descriptivo

En la Tabla 1, los ítems pertenecientes a las dimensiones de ira y miedo mostraron respuestas promedio a lo largo de todas sus categorías de respuestas con desviaciones contenidas. Contrariamente, en los ítems de ansiedad, miedo y depresión, se evidencia que el promedio de respuesta de los ítems presenta una tendencia hacia las alternativas de casi nunca y algunas veces. Además, el ítem 13. Siento que me falta el aire para respirar, perteneciente a la dimensión de Ansiedad, es quien muestra una mayor asimetría.

Tabla 1

Estadísticos descriptivos de los ítems del PIE-COVID19

\begin{tabular}{lcccc}
\hline \multicolumn{1}{c}{ Ítems } & $\mathbf{M}$ & $\mathbf{D E}$ & Asimetría & Curtosis \\
\hline Ira / Resentimiento & & & & \\
Ítem 01 & 1.62 & 0.97 & -0.04 & -1.04 \\
Ítem 02 & 1.62 & 0.87 & 0.02 & -0.77 \\
Ítem 03 & 1.63 & 0.89 & 0.11 & -0.89 \\
Ítem 04 & 1.54 & 0.97 & 0.01 & -1.01 \\
Ítem 05 & 2.27 & 0.89 & -0.96 & -0.09 \\
\hline Miedo & & & & \\
Ítem 06 & 1.33 & 0.86 & 0.30 & -0.55 \\
Ítem 07 & 1.89 & 0.88 & -0.24 & -0.89 \\
Ítem 08 & 1.46 & 0.96 & 0.03 & -0.97 \\
Ítem 09 & 1.99 & 0.87 & -0.59 & -0.32 \\
Ítem 10 & & 0.88 & -0.07 & -0.72 \\
\hline & & & & \\
\hline
\end{tabular}




\section{Ansiedad (por \\ Incertidumbre)}

Ítem 11

0.52

0.73

1.41

1.61

Ítem 12

0.85

0.94

0.93

$-0.04$

Ítem 13

0.30

0.61

2.15

4.55

Ítem 14

0.42

0.74

1.84

2.93

Ítem 15

0.45

0.77

1.68

2.05

\section{Estrés}

\begin{tabular}{ccccc} 
Ítem 16 & 0.93 & 0.85 & 0.55 & -0.50 \\
Ítem 17 & 1.07 & 0.98 & 0.57 & -0.71 \\
Ítem 18 & 0.92 & 0.95 & 0.77 & -0.38 \\
Ítem 19 & 0.98 & 0.99 & 0.73 & -0.54 \\
Ítem 20 & 0.89 & 0.90 & 0.82 & -0.08 \\
\hline Depresión & & & & \\
Ítem 21 & 0.66 & 0.89 & 1.23 & 0.64 \\
Ítem 22 & 0.96 & 0.89 & 0.75 & -0.12 \\
Ítem 23 & 0.58 & 0.84 & 1.40 & 1.19 \\
Ítem 24 & 0.63 & 0.85 & 1.33 & 1.08 \\
Ítem 25 & 0.70 & 0.83 & 1.13 & 0.74 \\
\hline & & & & \\
\hline & & & & \\
\hline
\end{tabular}

Análisis factorial confirmatorio y fiabilidad

Se desarrolló el AFC especificando el modelo teórico original del instrumento sin ninguna modificación o restricción adicional en el proceso de especificación. La 
evaluación del modelo de 5 factores fue excelente en todos sus índices $\left(X^{2}=426.83\right.$, $\mathrm{gl}=265, \mathrm{CFI}=.970, \mathrm{TLI}=.966, \mathrm{RMSEA}=.062, \mathrm{SRMR}=.079)$.

En la Tabla 2 se muestra las cargas factoriales del modelo evaluado. Todas las cargas mostradas son mayores a 0.63 y ascienden hasta 0.94. Adicionalmente, Los índices de fiabilidad estimados en todas las dimensiones son excelentes (>0.85).

Tabla 2

Cargas factoriales e índice de fiabilidad del modelo factorial del PIE-COVID19

\begin{tabular}{|c|c|c|c|c|c|}
\hline Ítems & $\lambda$ & [LI & - & LS] & Alfa y Omega \\
\hline \multicolumn{6}{|l|}{ Ira } \\
\hline Ítem 01 & 0.81 & 0.72 & - & 0.89 & \\
\hline Ítem 02 & 0.79 & 0.72 & - & 0.85 & \\
\hline Ítem 03 & 0.84 & 0.79 & - & 0.90 & $\begin{array}{l}\alpha=0.88 \\
\omega=0.86\end{array}$ \\
\hline Ítem 04 & 0.83 & 0.77 & - & 0.89 & \\
\hline Ítem 05 & 0.69 & 0.57 & - & 0.81 & \\
\hline \multicolumn{6}{|l|}{ Miedo } \\
\hline Ítem 06 & 0.88 & 0.82 & - & 0.93 & \\
\hline Ítem 07 & 0.90 & 0.86 & - & 0.94 & \\
\hline Ítem 08 & 0.85 & 0.80 & - & 0.90 & $\begin{array}{l}\alpha=0.90 \\
\omega=0.89\end{array}$ \\
\hline Ítem 09 & 0.94 & 0.90 & - & 0.98 & \\
\hline Ítem 10 & 0.63 & 0.54 & - & 0.73 & \\
\hline
\end{tabular}




\begin{tabular}{llllll}
\multicolumn{1}{l}{ Ansiedad } & & & & & \\
Ítem 11 & 0.82 & 0.72 & - & 0.92 & \\
Ítem 12 & 0.80 & 0.72 & -0.89 & \\
Ítem 13 & 0.72 & 0.60 & -0.84 & $\alpha=0.90$ \\
Ítem 14 & 0.82 & 0.74 & -0.91 & $\omega=0.85$ \\
Ítem 15 & 0.87 & 0.78 & -0.96 &
\end{tabular}

\section{Estrés}

$\begin{array}{llllll}\text { Ítem 16 } & 0.78 & 0.70 & -0.85 & \\ \text { Ítem 17 } & 0.86 & 0.81 & -0.92 & \\ \text { Ítem 18 } & 0.93 & 0.89 & -0.97 & \alpha=0.92 \\ & & & & & \omega=0.90\end{array}$

Ítem $19 \quad 0.84 \quad 0.77 \quad-\quad 0.91$

Ítem $20 \quad 0.85 \quad 0.79 \quad-\quad 0.91$

\section{Depresión}

$\begin{array}{llllll}\text { Ítem 21 } & 0.80 & 0.71 & -0.88 & \\ \text { Ítem 22 } & 0.84 & 0.78 & -0.90 & \\ \text { Ítem 23 } & 0.91 & 0.86 & -0.96 & \alpha=0.92 \\ & & & & & \omega=0.88\end{array}$

Ítem $24 \quad 0.87 \quad 0.81 \quad-\quad 0.93$

Ítem $25 \quad 0.79 \quad 0.71 \quad-\quad 0.87$

En la figura 1 se puede observar de igual manera las cargas factoriales de cada ítem con respecto a sus factores $y$, adicionalmente las correlaciones interfactoriales y la varianza específica de cada ítem (error). 


\section{Modelo Factorial Confirmatorio PIE - COVID - Perú}

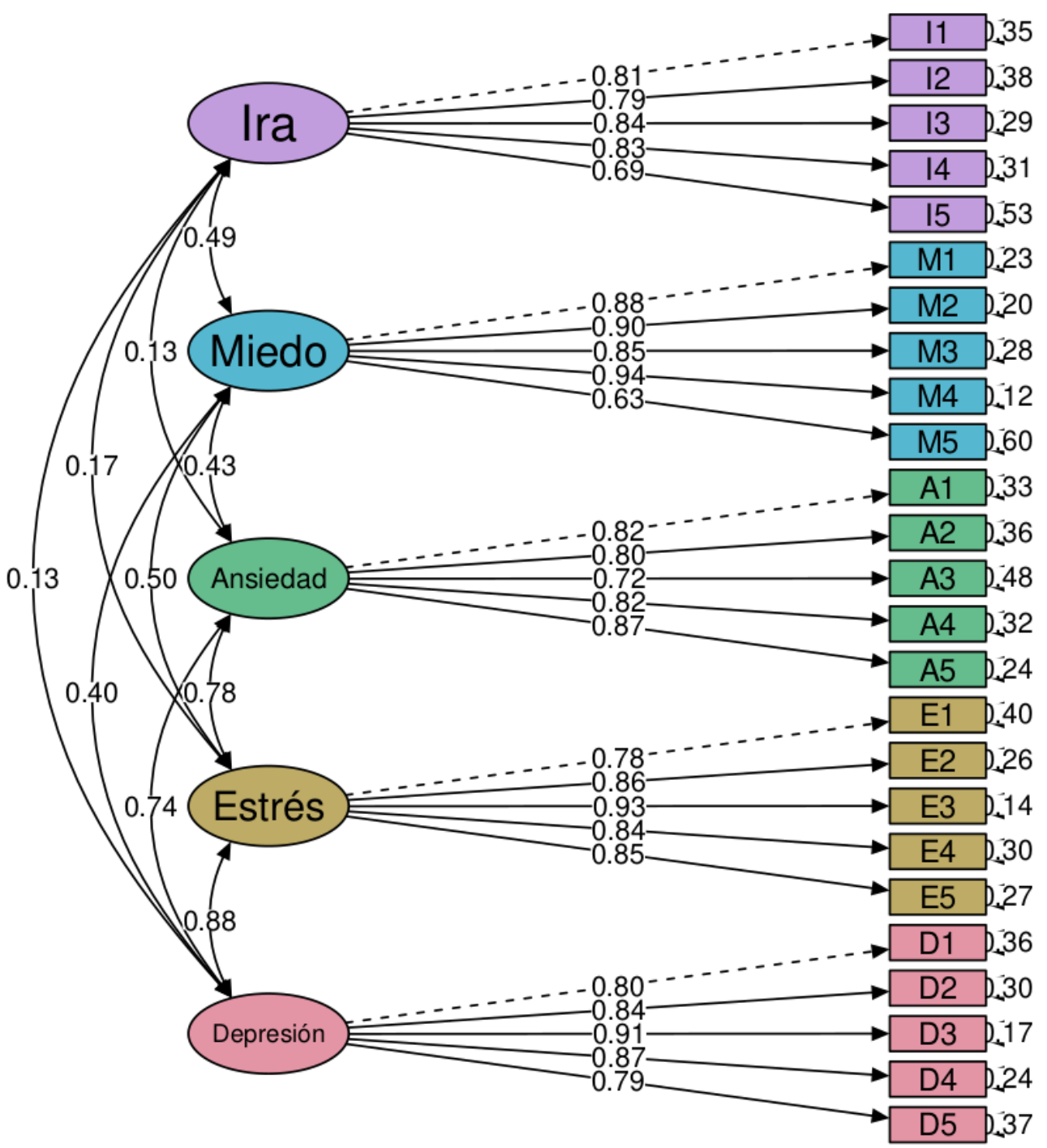

Figura 1. Modelo Factorial del PIE-COVID19 


\section{Discusión}

Los participantes han mostrado mayor reactividad ante los reactivos relacionados a la ira y el miedo con respecto al COVID-19, en contraste al resto de las dimensiones. Específicamente el ítem 05 "Me enfa o irrita pensar que algunas personas no actúen con responsabilidad y pongan en riesgo a los demás" es quien produce un mayor puntaje promedio, es decir, que la mayoría de las personas se posiciona entre a menudo y constantemente ante esta situación. Estos contenidos generalmente presentan un promedio alto de respuesta en la población (Olapegba et al., 2020).

Por el contrario los ítems relacionados con el factor ansiedad evidenciaron un promedio de respuestas menor con respecto al resto de factores. Específicamente el ítem 13 "En la actualidad siento que me falta el aire para respirar", mostró el menor promedio de respuesta positivo y a su vez una asimetría y curtosis elevada. Esto indica que al responder al ítem referido, la mayoría de personas concentró sus respuestas en la alternativa "Casi nunca".

El contenido del ítem 13, aunque obedece a una situación frecuente en episodios de ansiedad, también es uno de los principales síntomas del COVID-19 (Guan et al., 2020; Holshue et al., 2020), que además es de conocimiento y fácil identificación por la población general (Moghanibashi-Mansourieh, 2020). Esto podría generar un sesgo en las respuestas típicas esperables a ítems relacionados con ansiedad.

Por otro lado, el análisis de la estructura interna de la prueba (American Educational Research Association, American Psychological Association, \& National Council on Measurement in Education, 2014), es decir la correspondencia empírica entre la estructura teórica y los datos recolectados, mostró índices de ajustes excelentes en todas sus valores estimados (Keith, 2019). De esta manera, se tiene evidencia de validez de que las interpretaciones que se puedan realizar acerca de las puntuaciones recolectadas por el instrumento, sean válidas y correspondientes a lo teórico.

En esta misma línea, los ítems demostraron excelentes valores de carga factorial. Esto muestra que el rasgo que presenten las personas en cada uno de los factores, podrá ser recolectado adecuadamente con todos los ítems presentes en el instrumento. Este comportamiento es el esperado en una buena escala de medición. 
De esta forma, las estimaciones de la fiabilidad también muestran valores muy altos. Es decir que los ítems recogen respuestas consistentes de las personas a cada uno de los contenidos y objetivos propuestos. La cercanía entre los valores del coeficiente alfa y omega, es una evidencia de que los ítems guardan uniformidad en su importancia y calidad entre ellos.

En conclusión, el instrumento, en esta fase preliminar, muestra excelentes propiedades psicométricas en cuanto a su validez basado en estructura interna y sus estimaciones de fiabilidad. Se recomienda su uso en procesos de investigación relacionados con variables psicológicas y situación de COVID-19.

\section{Agradecimientos}

Agradecemos a Manuel García Pérez y Ángela Magaz Lago por la autorización para el uso del instrumento PIE-COVID19 utilizado en el presente estudio.

\section{Referencias}

American Educational Research Association, American Psychological Association, \& National Council on Measurement in Education. (2014). Standards for Educational and Psychological Testing. Washington, DC: American Educational Research Association.

Ashraf, B. N. (2020). Economic impact of government interventions during the COVID19 pandemic: International evidence from financial markets. Journal of Behavioral and Experimental Finance, 27, 100371. doi: 10.1016/j.jbef.2020.100371

Ato, M., López-García, J. J., \& Benavente, A. (2013). Un sistema de clasificación de los diseños de investigación en psicología. Anales de Psicología, 29(3), 1038- 
1059. doi: 10.6018/analesps.29.3.178511

Brooks, S. K., Webster, R. K., Smith, L. E., Woodland, L., Wessely, S., Greenberg, N., \& Rubin, G. J. (2020). The psychological impact of quarantine and how to reduce it: Rapid review of the evidence. The Lancet, 395(10227), 912-920. doi: $10.1016 / S 0140-6736(20) 30460-8$

Chau, C., \& Vilela, P. (2017). Determinantes de la salud mental en estudiantes universitarios de Lima y Huánuco. Revista de Psicología (PUCP), 35(2), 387422. doi: 10.18800/psico.201702.001

Cova Solar, F., Alvial S, W., Aro D, M., Bonifetti D, A., Hernández M, M., \& Rodríguez C, C. (2007). Problemas de Salud Mental en Estudiantes de la Universidad de Concepción. Terapia psicológica, 25(2), 105-112. doi: 10.4067/S071848082007000200001

Dubey, S., Biswas, P., Ghosh, R., Chatterjee, S., Dubey, M. J., Chatterjee, S., ... Lavie, C. J. (2020). Psychosocial impact of COVID-19. Diabetes \& Metabolic Syndrome: Clinical Research \& Reviews, 14(5), 779-788. doi: 10.1016/j.dsx.2020.05.035

Feldman, L., Goncalves, L., Chacón-Puignau, G., Zaragoza, J., Bagés, N., \& De Pablo, J. (2008). Relaciones entre estrés académico, apoyo social, salud mental y rendimiento académico en estudiantes universitarios venezolanos. Universitas Psychologica, 7(3), 739-752.

García Pérez, E. M., \& Magaz, A. (2020). PIE - COVID19: Perfil de Impacto Emocional de la COVID19. División de Investigación y Estudios Grupo ALBOR-COHS.

Guan, W., Ni, Z., Hu, Y., Liang, W., Ou, C., He, J., ... Zhong, N. (2020). Clinical Characteristics of Coronavirus Disease 2019 in China. New England Journal of Medicine. doi: 10.1056/NEJMoa2002032 
Guessoum, S. B., Lachal, J., Radjack, R., Carretier, E., Minassian, S., Benoit, L., \& Moro, M. R. (2020). Adolescent psychiatric disorders during the COVID-19 pandemic and lockdown. Psychiatry Research, 291, 113264. doi: 10.1016/j.psychres.2020.113264

Guo, Q., Zheng, Y., Shi, J., Wang, J., Li, G., Li, C., ... Yang, Z. (2020). Immediate psychological distress in quarantined patients with COVID-19 and its association with peripheral inflammation: A mixed-method study. Brain, Behavior, and Immunity. doi: 10.1016/j.bbi.2020.05.038

Gutiérrez Rodas, J. A., Montoya Vélez, L. P., Toro Isaza, B. E., Briñón Zapata, M. A., Rosas Restrepo, E., \& Salazar Quintero, L. E. (2010). Depression in university students and its association with academic stress. CES Medicina, 24(1), 7-17. Harapan, H., Itoh, N., Yufika, A., Winardi, W., Keam, S., Te, H., ... Mudatsir, M. (2020). Coronavirus disease 2019 (COVID-19): A literature review. Journal of Infection and Public Health, 13(5), 667-673. doi: 10.1016/j.jiph.2020.03.019

Holmes, E. A., O’Connor, R. C., Perry, V. H., Tracey, I., Wessely, S., Arseneault, L., ... Bullmore, E. (2020). Multidisciplinary research priorities for the COVID-19 pandemic: A call for action for mental health science. The Lancet Psychiatry, 7(6), 547-560. doi: 10.1016/S2215-0366(20)30168-1

Holshue, M. L., DeBolt, C., Lindquist, S., Lofy, K. H., Wiesman, J., Bruce, H., ... Pillai, S. K. (2020). First Case of 2019 Novel Coronavirus in the United States. New England Journal of Medicine. doi: 10.1056/NEJMoa2001191

Huamaní, C., Timaná-Ruiz, R., Pinedo, J., Pérez, J., \& Vásquez, L. (2020). Condiciones estimadas para controlar la pandemia de COVID-19 en escenarios de pre y poscuarentena en el Perú. Revista Peruana de Medicina Experimental y Salud Pública, 37(2), 195-202. doi: 


\subsection{3/rpmesp.2020.372.5405}

Huarcaya-Victoria, J. (2020). Consideraciones sobre la salud mental en la pandemia de COVID-19. Revista Peruana de Medicina Experimental y Salud Pública, 37(2), 327-334. doi: 10.17843/rpmesp.2020.372.5419

Keith, T. Z. (2019). Multiple regression and beyond: An introduction to multiple regression and structural equation modeling (Third Edition). New York: Routledge.

Lin, Q., Zhao, S., Gao, D., Lou, Y., Yang, S., Musa, S. S., ... He, D. (2020). A conceptual model for the coronavirus disease 2019 (COVID-19) outbreak in Wuhan, China with individual reaction and governmental action. International Journal of Infectious Diseases, 93, 211-216. doi: 10.1016/j.ijid.2020.02.058

Micin, S., \& Bagladi, V. (2011). Salud Mental en Estudiantes Universitarios: Incidencia de Psicopatología y Antecedentes de Conducta Suicida en Población que Acude a un Servicio de Salud Estudiantil. Terapia psicológica, 29(1), 53-64. doi: 10.4067/S0718-48082011000100006

Moghanibashi-Mansourieh, A. (2020). Assessing the anxiety level of Iranian general population during COVID-19 outbreak. Asian Journal of Psychiatry, 51, 102076. doi: 10.1016/j.ajp.2020.102076

Neponuceno, H. de J., Souza, B. D. M., Neves, N. M. B. C., Neponuceno, H. de J., Souza, B. D. M., \& Neves, N. M. B. C. (2019). Common mental disorders in medical students. Revista Bioética, 27(3), 465-470. doi: 10.1590/198380422019273330

Olapegba, P. O., Ayandele, O., Kolawole, S. O., Oguntayo, R., Gandi, J. C., Dangiwa, A. L., ... Iorfa, S. K. (2020). COVID-19 Knowledge and Perceptions in Nigeria [Preprint]. doi: 10.31234/osf.io/j356x 
Palacio Sañudo, J. E., Martínez de Biava, Y., Ochoa, N. L., \& Tirado Muñoz, E. (2006). Relación Del Rendimiento Académico Con Las Aptitudes Mentales, Salud Mental, Autoestima Y Relaciones De Amistad En Jóvenes Universitarios De Atlántico Y Bolívar. Psicogente, 9(15), 11-31.

Pappa, S., Ntella, V., Giannakas, T., Giannakoulis, V. G., Papoutsi, E., \& Katsaounou, P. (2020). Prevalence of depression, anxiety, and insomnia among healthcare workers during the COVID-19 pandemic: A systematic review and metaanalysis. Brain, Behavior, and Immunity. doi: 10.1016/j.bbi.2020.05.026

Perales, A., Izaguirre, M., Sánchez, E., Barahona, L., Martina, M., Amemiya, I., ... Padilla, A. (2019). Salud mental en estudiantes de pregrado de la Facultad de Medicina de la Universidad Nacional Mayor de San Marcos. Anales de la Facultad de Medicina, 80(4), 443-450. doi: 10.15381/anales.v80i4.17142

Qarnain, S. S., Muthuvel, S., \& Bathrinath, S. (2020). Review on government action plans to reduce energy consumption in buildings amid COVID-19 pandemic outbreak. Materials Today: Proceedings. doi: 10.1016/j.matpr.2020.04.723

Sánchez-Duque, J. A., Arce-Villalobos, L. R., \& Rodríguez-Morales, A. J. (2020). Enfermedad por coronavirus 2019 (COVID-19) en América Latina: Papel de la atención primaria en la preparación y respuesta. Atencion Primaria, 52(6), 369372. doi: 10.1016/j.aprim.2020.04.001

Shen, M., Peng, Z., Guo, Y., Rong, L., Li, Y., Xiao, Y., ... Zhang, L. (2020). Assessing the effects of metropolitan-wide quarantine on the spread of COVID-19 in public space and households. International Journal of Infectious Diseases, 96, 503505. doi: 10.1016/j.jijid.2020.05.019

Sivashanker, K., Mendu, M. L., Wickner, P., Hartley, T., Desai, S., Fiumara, K., ... Salmasian, H. (2020). Communication with Patients and Families Regarding 
Health Care-Associated Exposure to Coronavirus 2019: A Checklist to Facilitate Disclosure. The Joint Commission Journal on Quality and Patient Safety. doi: 10.1016/j.jcjq.2020.04.010

Suárez-Montes, N., \& Díaz-Subieta, L. B. (2015). Estrés académico, deserción y estrategias de retención de estudiantes en la educación superior. Revista de Salud Pública, 17, 300-313. doi: 10.15446/rsap.v17n2.52891

Troyer, E. A., Kohn, J. N., \& Hong, S. (2020). Are we facing a crashing wave of neuropsychiatric sequelae of COVID-19? Neuropsychiatric symptoms and potential immunologic mechanisms. Brain, Behavior, and Immunity, 87, 34-39. doi: 10.1016/j.bbi.2020.04.027

Wang, S., Wen, X., Liu, B., Dong, Y., \& Cui, M. hu. (2020). Psychological influence of Coronovirus disease 2019 (COVID-19) pandemic on the general public, medical workers and patients with mental disorders and its countermeasures. Psychosomatics. doi: 10.1016/j.psym.2020.05.005

World Health Organization. (2020). Situation Report-173 (N. ${ }^{\circ}$ 173). Recuperado de World Health Organization website: https://www.who.int/docs/defaultsource/coronaviruse/situation-reports/20200711-covid-19-sitrep173.pdf?sfvrsn=949920b4_2 
Educa UMCH. Revista sobre Educación y Sociedad, 2020, 1(15), 5-22. https://doi.org/10.35756/educaumch.v1i15.135 